\title{
Research highlights from the Ontario
} Veterinary College's Career

\section{Opportunities and Research Experience (CORE) Program}

\section{Overview}

This Special Series features research highlights from the Ontario Veterinary College's Career Opportunities and Research Experience (CORE) Program (previously the Summer Leadership and Research Program), which ran from May $5^{\text {th }}$ to August $12^{\text {th }}, 2016$. The CORE program was designed to provide B.SC and D.V.M. students with additional exposure to the professional world of veterinary medicine and research. Alongside their work with researchers and faculty members, student participants attended a Round Table series, several conferences, field trips to research facilities, and professional development workshops. CORE participants also delivered oral and poster presentations, sharing the results of their research with peers and with the University of Guelph's larger research community. Selected abstracts from these presentations are included here.

Keywords: Career Opportunities and Research Experience Program; CORE; Ontario Veterinary College; University of Guelph.

\section{The efficacy of the Revive Neo probiotic product on neonatal diarrhea in calves}

\section{Celeste Noble}

Faculty supervisors: Todd Duffield, David Renaud.

For correspondence, please email: cnoble01@mail.uoguelph.ca.

Diarrhea is the leading cause of death in pre-weaned dairy calves, and is frequently treated with antibiotics. As a potential alternative treatment, Revive Neo, a multi-species probiotic, is hypothesized to strengthen intestinal defenses by improving intestinal microbial balance. The objective of this triple-blind randomized controlled trial was to evaluate the efficacy of Revive Neo as a treatment for neonatal diarrhea in calves. On 10 dairy farms during the summer of 2016, calves observed with diarrhea were enrolled in the trial. Enrolled calves were randomly assigned to either the Revive Neo treatment group, or to the placebo treatment group (which were concealed as blue and red treatment groups, respectively, until the completion of trial). Time (days) until resolution of diarrhea, average daily gain (ADG) (lbs/day), and supportive therapies were monitored and recorded until weaning. A total of 157 calves were enrolled, with 82 in the blue group, and 75 in the red group. Two linear regression models were created to examine the impact of each treatment on ADG in the first week after enrollment, and the number of days to resolution of abnormal fecal score. Treatment in the red group significantly decreased the number of days to resolution of abnormal fecal score, reducing it by 2.11 days if the calf was enrolled after 13 days of age ( $p=0.037$ ). However, no significant difference in ADG was found between treatment groups in the first week after enrollment ( $p=0.626$ ). These results form part of an ongoing trial which may, when complete, yield a clearer understanding of the efficacy of Revive Neo as a treatment for neonatal diarrhea in calves.

\section{Developing a clonality assay to diagnose equine $\mathrm{T}$-cell lymphoma in the T-cell receptor gamma locus}

\author{
Elizabeth Silk \\ Faculty Supervisor: Ste fan M. Keller. \\ For corres pondence, please email: esilk@mail.uoguelph.ca.
}

Lymphoma is the most common hematopoietic tumor in horses, with an incidence rate between 1.3-2.8\%. Current diagnostic methods, which include clinical assessment and pathological evaluation of cell or tissue samples, are often inadequate for differentiating lymphoma from reactive conditions, owing to overlapping cellular morphology. Clonality testing, a genetic method used to diagnose lymphoma, has been established for use in dogs and cats, but 
not for use in horses. Clonality testing utilizes lymphocyte antigen receptor gene diversity to infer the clonality of lymphocytes in a diagnostic sample. This project sought to develop a clonality assay for the diagnosis of equine T-cell lymphoma in the equine T-cell receptor gamma (TRG) locus. A highly-multiplexed primer set covering all possible TRG rearrangements was developed. Cycling conditions were optimized for conventional polymerase chain reaction (PCR), followed by size separation of amplicons via capillary electrophoresis. To determine assay sensitivity and specificity, 10 neoplastic and 18 normal lymphoid samples were assessed with the novel assay. Of the samples, three of $10(30 \%)$ neoplastic samples and six of 18 (33.3\%) normal samples yielded clear-cut clonal and polyclonal electrophoresis patterns, respectively. In seven of $10(70 \%)$ neoplastic samples, variably distinct bands were accompanied by a polyclonal background. Unexpectedly, variably distinct bands were also seen in 12 of 18 (66.6\%) normal lymphoid samples, making them virtually indistinguishable from neoplastic samples with a polyclonal background. The nature of these variably distinct bands remains unclear, but it is assumed that they reflect low TRG diversity. This phenomenon increases the probability of false positive results, making the equine TRG locus an unreliable target for clonality testing in horses.

\section{Optimizing DNA extraction and quantitation for low cellularity samples in dogs}

\section{Akash Jairaj, Jodi Morrison}

Fa culty Supervisors: Fiona M.K. Ja mes, Stefan M. Keller. For correspondence, please email: jairaja@mail.uoguelph.ca.

Clonality testing is a molecular method used to diagnose lymphoma. For suspected central nervous system lymphomas, cerebrospinal fluid (CSF) is the only available sample type. However, CSF samples have relatively low volume and cellularity, and, in veterinary medicine, clonality testing has not yet been established for this sample type. The objective of this study was to determine suitable methods of DNA extraction and quantitation for low cellularity samples in dogs. A dilution series of the canine B-cell lymphoma cell line, CLBL-1, was used as a substitute for CSF samples. The efficiency of four DNA extraction methods (Qiagen QiaAMP Micro DNA Kit, GeneAid Geneius Micro gDNA Kit, Epicentre QuickExtract Solution, and a non-commercial protocol) were evaluated using three different quantitation methods. These were quantitative polymerase chain reaction (qPCR) (LC480), fluorometry (Qubit) and spectrophotometry (Nanodrop). Clonality testing was performed on all extractions. The lowest dilutions that yielded detectable DNA were 10,000 cells for the Qiagen Kit and the non-commercial protocol, and 100,000 cells for the GeneAid Kit. The lower detection limits of quantitation for qPCR and fluorometry were 1,000 cells and 10,000 cells, respectively. For spectrophotometry, the limit was undetermined. Clonality PCRs yielded successful amplification of DNA extracted from 100,000 cells using the GeneAid Kit, and from 10,000 cells using the Qiagen Kit and the non-commercial protocol. The lower efficiency of the Epicentre Kit may in part be due to its higher elution volume. This study identified suitable DNA extraction and detection methods for low cellularity samples, and these data provide the basis for clonality testing on CSF samples in dogs.

\section{Extended-spectrum cephalosporin resistance in canine fecal Enterobacteriaceae in Southern Ontario}

\author{
Xiao Shen, Pauline Zhang \\ Faculty Supervisor: Pa trick Boerlin. \\ For correspondence, please email:xshen04@mail.uoguelph.ca.
}

Extended-spectrum cephalosporins (ESCs) are important antimicrobials used in both human and veterinary medicine for treating serious bacterial infections. Their effectiveness, however, has been greatly threatened by rapidly emerging antimicrobial resistant (AMR) bacteria, many of which belong to the Enterobacteriaceae family. These Gram-negative bacteria inhabit the intestinal tract. They are pathogenic or opportunistic pathogens, and are commonly resistant to ESCs. Transfer of ESC-resistant Enterobacteriaceae occurs through contact between hosts. Resistance genes on plasmids are also exchanged between bacteria. This project investigated the prevalence of ESCresistant fecal Enterobacteriaceae and associated resistance genes in dogs in Southern Ontario. Fecal samples were collected from dog parks in 10 cities in Southern Ontario and were enriched with Enterobacteriaceae-selective media containing ESCs. The resulting ESC-resistant isolates were identified by MALDI-TOF (matrix assisted laser desorption/ionization-time of flight) mass spectrometry. PCR (polymerase chain reaction) was then performed to screen the isolates for the ESC-resistance genes bla $a_{\mathrm{CMY}}$, bla $a_{\mathrm{CTX}-\mathrm{M}}$, bla $_{\mathrm{SHV}}$, and bla OXA. Of the 234 samples, 28.6\% $(\mathrm{n}=67)$ carried ESC-resistant Enterobacteriaceae. Ninety-one unique isolates were identified, of which $89 \%$ were Escherichia coli. Of the isolates, 54 carried the AmpC-like $\beta$-lactamases gene bla $a_{\mathrm{CMY}}$, and 19 carried bla $a_{\mathrm{CTX}-\mathrm{M}}$, an emerging type of extended-spectrum $\beta$-lactamases. Sequencing revealed variants CTX-M-14 and CTX-M-27, which are commonly reported in human isolates. The CTX-M-1 variant, widespread in human and animal isolates in Europe, was also found. In Canada, however, the CTX-M-1 variant has previously only been isolated from bacteria in chickens. These results suggest that dogs can receive ESC-resistant Enterobacteriaceae from humans, possibly through fecal-oral and environmental routes, or through raw diets that include chicken. 


\section{Mortality and the Ontario Racehorse}

\section{Amanda Jowett}

Fa culty Supervisor: Peter Phys ick-Sheard.

For correspondence, please email: ajowett@mail.uoguelph.ca.

Racehorse mortality is an equine welfare issue, and one which additionally carries economic implications, poses safety risks for riders and drivers, and exerts a substantial negative impact on public perceptions of the racing industry. Available statistics concerning racehorse mortality vary by racing jurisdiction and lack consistent definition. Reliable measures of mortality are required in Ontario, and are available through Ontario Racing Commission's Death Registry. This study uses the Death Registry to quantify mortality rates for racehorses in Ontario, using clearly stated definitions to describe factors associated with mortality, and to identify possible underlying causes. The inclusion in the Death Registry of any instance of mortality within 60 days of a race provides a more consistent measure of racehorse mortality than previous studies have been able to achieve. There were 1,349 racing-associated instances of racehorse mortality in Ontario between the years 2003 and 2011. The overall mortality rate was 0.85 per 1,000 race starts. The mortality rate declined across the study period, and was influenced by age and sex. The mortality rate for an intact male was twice that for a gelding or mare. Moreover, the mortality rate for Thoroughbred racehorses was strongly influenced by age, and was 5.35 times the rate for Standardbreds. The most common presenting complaints were musculoskeletal injury, sudden unexplained death, and colic. Although negative publicity primarily reflects mortality that occurs during live races, these deaths represented less than $6 \%$ of all instances of mortality. This project is ongoing and uses statistical modelling to identify factors associated with mortality and to provide insight into causes.

\section{The efficacy of case-based assignments in teaching veterinary ophthalmology}

\section{Claire Segeren}

Fa culty supervisors: Cha ntale Pinard, Jennifer Reniers, Dale

Lackeyram.

For correspondence, please email:cs egeren@mail.uoguelph.ca.

Veterinarians approach clinical cases by first assessing symptoms, and subsequently reasoning toward a diagnosis and an appropriate treatment plan. Previous studies have demonstrated that case-based assignments can be used as effective tools for teaching these problem-solving skills and approaches in clinical programs. Very few studies, however, have focused on veterinary medicine or specializations within the field. This study aimed to assess the validity of the online case-based assignments given to students during the Ontario Veterinary College's (OVC) Phase IV Ophthalmology rotation from 2011 to 2016. These cases provided students with histories and images of small animal patients. With this information, students were asked to describe ocular lesions and to provide an appropriate diagnosis, a short-term treatment plan, and a long-term treatment plan. A rubric was developed to reflect the cognitive progression used when solving clinical problems. A frequency analysis was conducted to evaluate final grades across each case, and grades for each question across all cases. A total of 283 students were graded individually. Students' grades were normally distributed across each assignment and each question, indicating that all cases had the appropriate structure. Grade distribution displayed a rightward shift for questions requiring a treatment plan, indicating that there was an impediment in employing skills of analysis and evaluation. Our results appear to validate the use of these cases. Our future research aims to use both quantitative and qualitative data to assess whether the casebased assignments have a positive effect on students' learning in the OVC's Phase IV Ophthalmology rotation. 